not complete, understanding of the effects of alcohol consumption on the liver. Clinical and histological features of alcoholic liver disease (ALD) are used to assist clinicians in diagnosing and determining the severity of disease in patients with ALD. In this study, we provide salient points on their clinical and histological features. In addition, the benefits of liver biopsy and important differential diagnoses, including features distinguishing these entities.

Aims This study intends to describe in detail the clinical and histological features of ALD.

Methods Liver biopsy was required for the diagnosis of histologic ALD. A total of 83 patients with ALD were included in the study. Evaluate the stage of liver fibrosis according to Metavir classification (F0,F1,F2,F3,F4). Clinical and laboratory parameters were recorded.

Results The average age was $51.41 \pm 9.69$ years, the age group of 40-49 accounted for $43.4 \%$. 83 patients included in the study were males. Clinical symptoms were hepatomegaly (62.7\%), jaundice (67.5\%). Histological results were mostly fatty liver (92.8\%), significant fibrosis ( $\mathrm{F} \geq \mathrm{F} 2)$ accounted for $63.9 \%$, foamy degeneration accounted for $84.3 \%$, megamitochondria accounted for $63.4 \%$ and Mallory bodies accounted for $60.2 \%$.

Conclusions Patients in working age were often common. Common clinical symptoms were hepatomegaly and jaundice. The patients had salient points on histological features of ALD such as foamy degeneration, megamitochondria, Mallory body, fatty liver, significant fibrosis liver, widespread neutrophilic infiltration. Liver biopsy played an important role in the real-time clinical assessment of patients. Tissue histology not only yields diagnostic information but also important information about the patient's overall disease progression.

\section{IDDF2018-ABS-0031 THE CLINICAL FEATURES OF HBSAG AND ANTIHBC (+) HEPATOCELLULAR CARCINOMA IN HEPATITIS B}

Patricia Te*, Stephen Wong Chinese. General Hospital and Medical Center, Philippines

\subsection{6/gutjnl-2018-IDDFabstracts. 193}

Background Hepatocellular carcinoma (HCC) is the second most common cancer in the Philippines. The most common risk factor for HCC is hepatitis B and C. We investigated the clinical characteristics of HCC with past HBV and correlated it with HBsAg.

Methods Records of HCC patients with viral hepatitis in aetiology for 7 years were analysed. They were then divided into $\mathrm{HBsAg}(+) / \operatorname{antiHBC}(+)$ and $\mathrm{HBsAg}(-) / \operatorname{antiHBC}(+)$. The two groups were compared using T-test and Chi-square for continuous and categorical variables. SPSS version 20 was employed for all analysis.

Results Among 207 patients, HBsAg +/antiHBc +accounted for $43.48 \%$ of the cases, while HBsAg -/antiHBc $+56.52 \%$. The average age of presentation was in the 5 th to 6 th decade of life, with HBsAg (-) being affected at an older age (66.88 $\pm 11.454 ; \mathrm{p}=0.003$ ). Men were mostly afflicted. HCC from hepatitis $\mathrm{C}$ were significantly associated with $\mathrm{HBsAg}-/ \mathrm{antiHBc}$ $+\quad(\mathrm{p}=0.004)$. Only INR was statistically significant at $\mathrm{p}$ 0.041 , with HBsAg (+) having a higher INR at 1.22 vs 1.12 $(\mathrm{p}=0.041)$.

\begin{tabular}{lccc}
\multicolumn{4}{l}{ Abstract IDDF2018-ABS-0031 Table 1} \\
& $\begin{array}{l}\text { HBsAg }+ \text { /antiHBc }+ \\
43.48 \%(n=90)\end{array}$ & $\begin{array}{c}\text { HBsAg -/antiHBc }+ \\
56.52 \%(n=117)\end{array}$ & $p$ value \\
\hline Age $n=194$ & $59.86 \pm 12.843$ & $66.88 \pm 11.454$ & 0.000 \\
Gender $n=207$ & & & 0.380 \\
Female & $11.1 \%(n=10)$ & $16.4 \%(n=19)$ & \\
Male & $88.9 \%(n=80)$ & $83.6 \%(n=97)$ & \\
Family history $n=207$ & $8.89 \%(n=8)$ & $8.55 \%(n=10)$ & 1.000 \\
BMI $n=207$ & & & 0.772 \\
Under & $1.8 \%(n=1)$ & $4.22 \%(n=3)$ & \\
Healthy & $49 \%(n=27)$ & $40.8 \%(n=29)$ & \\
Preobese & $27.3 \%(n=15)$ & $25.35 \%(n=18)$ & \\
Obese & $21.8 \%(n=12)$ & $29.6 \%(n=21)$ & \\
Comorbid $n=207$ & & & \\
Hypertension & $15.56 \%(n=14)$ & $5.18 \%(n=6)$ & \\
Diabetes & $16.67 \%(n=15)$ & $24.78 \%(n=29)$ & \\
\hline
\end{tabular}

Conclusions The prevalence of antiHBc HCC is common in the Philippines and is more common than HCV related HCC. There are some differences in the clinical characteristics between HBsAg (+) and (-) groups, but this might be due to the first exposure to the virus. It is suggested that the natural course of hepatitis B and the risk for HCC be reconsidered. HCV also contribute to the risk of HCC despite having HBsAg seroclearance.

\section{IDDF2018-ABS-0033 ASSESSMENT OF LIVER FIBROSIS USING FIBROSCAN IN PATIENTS WITH ALCOHOLIC LIVER DISEASE}

Le Thi Thu Hien*. Internal Medicine, University of Medicine and Pharmacy, Thai Nguyen, Vietnam

\subsection{6/gutjnl-2018-IDDFabstracts. 194}

Background Alcoholic liver disease (ALD) is the most common cause of liver cirrhosis in the Western world. Systematic screening for liver fibrosis in heavy-drinking patients is a challenge. Liver biopsy is currently considered the gold standard for assessing hepatic fibrosis or cirrhosis in these patients. However, it is an invasive procedure, with rare but potentially life-threatening complications, assessing fibrosis is limited owing to sampling error. Fibroscan is a novel rapid and noninvasive method to assess liver fibrosis via liver stiffness (LS). LS was shown to be strongly associated with the degree of liver fibrosis in all of these patients.

Aims This study was to assess the accuracy of LS measurement for the diagnosis of extensive liver fibrosis in patients with ALD and to determine diagnostic LS cut-off values.

Methods 93 inpatients with ALD underwent concomitant Ultrasound-Guided liver biopsy (figure 1,2,3,4) and Fibroscan (figure 5), in 103 Military Hospital from January 2016 to November 2017. Fibrosis was evaluated according to the METAVIR system as validated in ALD for fibrosis (F0: absence of fibrosis, F1: minimal portal fibrosis without septa, F2: portal fibrosis with a few septa, F3: septal fibrosis, F4: cirrhosis). LS cut-offs were determined using receiver-operating characteristic (ROC) curves. 